\title{
Diferenciais intra-urbanos no Município de Florianópolis, Santa Catarina, Brasil: potencial de uso para o planejamento em saúde
}

\author{
Intra-urban differentials in Florianópolis, \\ Santa Catarina State, Brazil, and their potential \\ use in health services planning
}

Josimari Telino de Lacerda 1

Maria Cristina Marino Calvo 2

Sérgio Fernando Torres de Freitas 2

\footnotetext{
1 Universidade do Sul de Santa Catarina. Av. José Acácio Moreira 787 Tubarão, SC

88704-900, Brasil. jtelino@terra.com.br 2 Departamento de Saúde Pública, Centro de Ciências da Saúde, Universidade Federal de Santa Catarina. Campus Universitário $s / n$, Florianópolis, SC 88040-900, Brasil. mcmcalvo@terra.com.br storres@repensul.ufsc.br
}

\begin{abstract}
Based on the premise that spatial occupation by the population is a socially constructed process, the present study identified and grouped homogeneous sectors vis-à-vis living conditions in the municipality of Florianópolis, Santa Catarina State, Brazil. Among socioeconomic variables from the Brazilian national census, the study identified and selected those which best represent the intra-urban differentials in living conditions in Florianópolis. The variables were categorized according to uniformity in the groups and orientation from the literature. Factor correspondence analysis was used for sector grouping. High income, low income, high schooling, illiteracy, and sub-standard garbage collection were the variables used to define grouping. As a result, the authors were able to divide the municipality into four homogeneous groups: two opposite groups having the best and worst schooling and income, and two median groups in which access to public services defines the hierarchical level. The northern and southern regions of Florianópolis were identified as receiving the greatest attention from the city administration. A heterogeneous distribution of households within sectors was identified, whereby Florianópolis was characterized as not presenting large agglomerations of poverty; however, this should not be interpreted as the absence of a poor population in the municipality.
\end{abstract}

Key words Factor Analysis; Living Conditions; Residence Characteristics; City Planning

Resumo Partindo do pressuposto de que a ocupação espacial da população é um processo socialmente construído, e que o espaço social é determinante no processo saúdeldoença, o presente estudo identificou e agrupou os setores homogêneos quanto à condição de vida no Município de Florianópolis. Selecionou, dentre as variáveis sócio-econômicas do censo demográfico brasileiro, as que melhor identificavam os diferenciais intra-urbanos de condição de vida no município. Categorizou as variáveis observando os aspectos de uniformidade dos grupos e a orientação da literatura. Utilizou a Análise Fatorial de Correspondência, no processo de agrupamento dos setores, definido pelas variáveis: Renda Alta, Renda Baixa, Escolaridade Alta, Analfabetismo e Coleta Precária do Lixo. Como resultado, observou que o município pode ser dividido em quatro grupos homogêneos: dois oponentes, com melhores e piores condições de escolaridade e renda e dois medianos, nos quais a diferença é determinada pelo acesso aos serviços públicos. Indica as regiões Norte e Sul como as merecedoras de maior atenção dos gestores públicos. Identifica uma distribuição heterogênea dos domicílios no interior dos setores, caracterizando Florianópolis por não apresentar grupos concentrados de pobreza, o que não pode ser entendido como ausência desses grupos no município. Palavras-chave Análise Fatorial; Condições de Vida; Distribuição Espacial; Planejamento Urbano 


\section{Introdução}

O conhecimento dos determinantes, da distribuição e da freqüência dos agravos de saúde na coletividade é primordial para os gestores dos serviços de saúde, o que faz da epidemiologia ciência básica na área da Saúde Pública. Atua como propulsora do desenvolvimento de tecnologias efetivas ao estudo do processo saúde/doença na sociedade, fornecendo subsídios ao controle, prevenção e erradicação dos agravos à saúde. Dentre esses subsídios, destaca-se a identificação dos grupos e áreas de maior risco, atuando como suporte básico do planejamento local dos serviços (Almeida Filho \& Rouquayrol, 1992; Pereira, 1995).

Organismos internacionais especializados e a comunidade científica, preocupados com as desigualdades de saúde da população, apontam a necessidade do desenvolvimento de projetos que contemplem ações integrais, multissetoriais, não limitadas à atenção médica, para transformar o quadro de saúde da população.

Uma das estratégias adotadas pelos serviços de saúde pública para viabilizar tais metas, tem sido a implantação dos Sistemas Locais de Saúde (SILOS), nos quais as Unidades de Saúde são responsáveis por uma população adscrita num território, e onde o planejamento das ações deve se pautar nas necessidades da comunidade local. O pressuposto básico dessa concepção de organização dos serviços é que as enfermidades e os agravos de saúde estão espacialmente distribuídos, de modo particular, a cada grupo populacional.

A compreensão dos diferenciais de saúde entre os grupos sociais e dentro de cada grupo torna-se ponto crucial nos estudos epidemiológicos dessa linha de pensamento. Autores como Paim (1997) e Castellanos (1992), ressaltam a importância do espaço social nos estudos de explicação do processo saúde/doença. Argumentam que se o processo saúde/doença pode ser compreendido como resultante da posição dos indivíduos no espaço social e das relações daí decorrentes, o estudo desse espaço tornase fundamental no campo da epidemiologia.

A localização geográfica dos grupos de maior prevalência ou risco das doenças tem sido motivo de inquietação de vários estudiosos. Em sua revisão sobre os métodos utilizados nos estudos de desigualdades sociais, Borrell (1997), descreve os principais indicadores utilizados nos trabalhos, quais sejam: classe social, nível de escolaridade, ocupação, renda, além dos indicadores que caracterizam o lar. Locker (1993), apresenta algumas limitações das medidas mais convencionais de iniqüidade social: renda, educação e ocupação. Segundo o autor, esses indicadores estando relacionados a dados individuais ou de familiares, não têm o potencial de identificar grupos homogêneos, não estão voltados ao meio em que as pessoas vivem, sendo, portanto, insuficientes para a localização espacial dos mesmos. Apresenta como alternativa as "area-based measures" que utilizam dados sócio-econômicos das regiões em que vivem as pessoas. Tais medidas, caracterizadas como indicadores compostos, combinam vários fatores sócio-econômicos como referenciais para a descrição de áreas geográficas, pressupondo que essa combinação reproduz a interação dos diversos fatores determinantes da qualidade de vida daquela área.

O desenvolvimento de métodos diagnósticos coletivos, com o potencial de discernimento de grupos ou áreas de risco no interior da coletividade, e que sirvam de instrumento ao planejamento dos serviços de saúde, tem sido um grande desafio para os epidemiologistas e planejadores do setor. O cadastramento das famílias e usuários tem sido a ferramenta utilizada no Brasil, em especial nos Programas de Saúde da Família (PSF) e Agentes Comunitários de Saúde (PACS). A coleta de informações sociais, comportamentais e de antecedentes familiares realizada pelas equipes de saúde, tem por finalidade última a identificação de grupos e/ou áreas de risco, sobre os quais definirão suas prioridades. Entretanto, os estudos científicos relatados anteriormente, indicam a possibilidade do estabelecimento dessas áreas e/ou grupos, através de dados existentes. No Brasil, o Censo Demográfico Decenal da Fundação Instituto Brasileiro de Geografia e Estatística (IBGE), constitui-se em fonte oficial de dados sociais disponível a todos os municípios, com mapeamento e identificação geográfica das informações.

Uma reflexão diante desses pressupostos e informações motivou o objetivo geral deste trabalho, que é identificar grupos e/ou áreas semelhantes em suas condições de vida, a partir dos dados do Censo do IBGE.

A caracterização de áreas pode ser um instrumento útil para ações de planejamento, especialmente para a saúde, já que áreas homogêneas podem ser assumidas como similares em sua situação de morbidade.

\section{Materiais e métodos}

A unidade de análise do estudo - setor censitário - é a unidade territorial de controle cadastral de coleta utilizada pelo IBGE na realização 
do censo demográfico. Florianópolis está dividida em 297 setores, agregados em dez distritos. Cada setor compreende um total de 250 a 300 domicílios. No relatório do IBGE do Censo de 1991 (indicadores sócio-econômicos), observou-se ausência parcial ou total de informações em seis setores, que foram excluídos do estudo (IBGE, 1991).

O processo de análise e seleção dos melhores representantes dos diferenciais intra-urbanos do município incluiu todas as categorias sócio-demográficas coletadas pelo IBGE. O processo de categorização das áreas homogêneas quanto a Condição de Vida no município de Florianópolis, obedeceu três etapas relatadas a seguir.

\section{Seleção das variáveis}

Dentre as variáveis sócio-econômicas coletadas pelo IBGE, foram selecionadas as que melhor identificaram os diferenciais intra-urbanos do município, diretamente relacionadas com Condição de Vida, quais sejam: de saneamento básico (abastecimento de água, esgoto e destino do lixo), escolaridade e renda do chefe da família.

Outras variáveis apontadas na literatura como importantes na análise de Condição de Vida - disponíveis, mas não utilizadas neste estudo - são a condição de habitação, em especial a aglomeração domiciliar, e a condição de propriedade da habitação. A aglomeração domiciliar descrita por Locker (1993), é definida pela taxa de pessoa por cômodo apropriado para dormir - quarto e sala. Na classificação de condição de habitação, a Fundação Sistema Estadual de Análise de Dados (Fundação Seade, 1992c), adota como parâmetro satisfatório dos domicílios, a presença de no mínimo quatro cômodos (uma sala, um quarto, uma cozinha e um banheiro), sem que haja superposição de funções nos cômodos, ou seja, onde sala e cozinha não estejam sendo utilizados como dormitório.

Para a coleta de dados do IBGE, cômodo é toda e qualquer dependência domiciliar (quarto, sala, banheiro, cozinha, área de serviço e garagem), e seu relatório não propicia a identificação dos tipos de cômodos, nem sua função no domicílio. Assim, a informação sobre aglomeração ou satisfação dos domicílios, como descrita na literatura, fica prejudicada com os dados disponíveis. Além disso, em Florianópolis, apenas $2,7 \%$ dos setores apresentavam em média menos de quatro cômodos por domicílio. Com essas constatações, a variável "cômodo por domicílio” foi excluída do estudo.
A análise da condição de propriedade evidenciou que cerca de $75 \%$ da população é proprietária de seus domicílios. A formação de aglomerações de famílias de baixa renda em Florianópolis, tem se construído a partir de invasões de áreas públicas não recuperadas e assumidas como propriedade pela população, e por doação de propriedade nas áreas mais carentes do município pela administração local. Assim, apropriação de moradia não expressa variabilidade da Condição de Vida em Florianópolis, não sendo utilizada como diferencial intra-urbano no estudo.

\section{Categorização das variáveis}

Os setores censitários foram analisados quanto aos percentuais de domicílios com presença das condições estudadas, constituindo três categorias: setor com baixa, média e alta presença da condição. As faixas percentuais que definiram as categorias, foram estabelecidas segundo Horber \& Ladiray (1997), onde as categorias de variáveis devem conter número de elementos semelhantes, para evitar que grupos pequenos tenham maior peso na análise.

A Tabela 1 apresenta as variáveis selecionadas e suas categorias.

Para as condições de saneamento, foram consideradas as presenças de: abastecimento de água tratada, esgotamento sanitário ou fossa séptica e coleta de lixo regular.

A condição de escolaridade foi categorizada em baixa, média e alta presença de analfabetos, menos de 8 anos de escolaridade e mais de 8 anos de escolaridade. Esses parâmetros estão baseados nos estudos da Fundação Seade (1992a), entendendo que Menos de 8 anos coloca os indivíduos aí inseridos num patamar inferior ao mínimo de conhecimentos básico e elementar exigidos pela Constituição enquanto direito civil, indicando um nível inferior de acesso à informação, refletindo-se na Condição de Vida.

A condição de renda foi igualmente subdividida em três categorias: baixa, média e alta presença de domicílios nas faixas até 2 salários mínimos, de 2 a 6 salários mínimos e acima de 6 salários mínimos. Entende-se que no primeiro parâmetro encontram-se as famílias no limite da pobreza, segundo a Fundação Seade (1992b) e Jaguaribe et al. (1986). O segundo ponto de corte foi definido a partir da análise da distribuição da variável renda, a qual indicou que o limite acima de 6 salários mínimos expressava melhor os diferenciais intra-urbanos no município. 
Tabela 1

\begin{tabular}{|c|c|c|c|}
\hline Variáveis (presença de) & Categoria 1 & Categoria 2 & Categoria 3 \\
\hline Água de abastecimento público & $\begin{array}{l}\text { AG1: Baixa } \\
\text { Até } 30 \% \text { dos domicílios }\end{array}$ & $\begin{array}{l}\text { AG2: Média } \\
\text { De } 30 \text { a } 80 \%\end{array}$ & $\begin{array}{l}\text { AG3: Alta } \\
\text { Mais de } 80 \%\end{array}$ \\
\hline Esgoto adequado & $\begin{array}{l}\text { EG1: Baixa } \\
\text { Até } 50 \% \text { dos domicílios }\end{array}$ & $\begin{array}{l}\text { EG2: Média } \\
\text { De } 50 \text { a } 80 \%\end{array}$ & $\begin{array}{l}\text { EG3: Alta } \\
\text { Mais de } 80 \%\end{array}$ \\
\hline Coleta de lixo & $\begin{array}{l}\text { LX1: Baixa } \\
\text { Até } 50 \% \text { dos domicílios }\end{array}$ & $\begin{array}{l}\text { LX2: Média } \\
\text { De } 50 \text { a } 80 \%\end{array}$ & $\begin{array}{l}\text { LX3: Alta } \\
\text { Mais de } 80 \%\end{array}$ \\
\hline Analfabetismo & $\begin{array}{l}\text { AN1: Baixa } \\
\text { Até } 15 \% \text { de analfabetos }\end{array}$ & $\begin{array}{l}\text { AN2: Média } \\
\text { De } 15 \text { a } 50 \%\end{array}$ & $\begin{array}{l}\text { AN3: Alta } \\
\text { Mais de } 50 \%\end{array}$ \\
\hline $\begin{array}{l}\text { Escolaridade baixa } \\
\text { (menos de } 8 \text { anos de estudo) }\end{array}$ & $\begin{array}{l}\text { EB1: Baixa } \\
\text { Até } 15 \% \text { dos chefes } \\
\text { de família }\end{array}$ & $\begin{array}{l}\text { EB2: Média } \\
\text { De } 15 \text { a } 50 \% \text { dos chefes } \\
\text { de família }\end{array}$ & $\begin{array}{l}\text { EB3:Alta } \\
\text { Mais de } 50 \%\end{array}$ \\
\hline $\begin{array}{l}\text { Escolaridade alta } \\
\text { (mais de } 8 \text { anos de estudo) }\end{array}$ & $\begin{array}{l}\text { EA1: Baixa } \\
\text { Até } 15 \% \text { dos chefes } \\
\text { de família }\end{array}$ & $\begin{array}{l}\text { EA2: Média } \\
\text { De } 15 \text { a } 50 \% \text { dos chefes } \\
\text { de família }\end{array}$ & $\begin{array}{l}\text { EA3: Alta } \\
\text { Mais de } 50 \%\end{array}$ \\
\hline $\begin{array}{l}\text { Renda baixa } \\
\text { (até } 2 \text { salários mínimos) }\end{array}$ & $\begin{array}{l}\text { RB1: Baixa } \\
\text { Até } 20 \% \text { dos chefes }\end{array}$ & $\begin{array}{l}\text { RB2: Média } \\
\text { De } 20 \text { a } 50 \%\end{array}$ & $\begin{array}{l}\text { RB3: Alta } \\
\text { Mais de } 50 \%\end{array}$ \\
\hline $\begin{array}{l}\text { Renda mediana } \\
\text { (de } 2 \text { a } 6 \text { salários mínimos) }\end{array}$ & $\begin{array}{l}\text { RM1: Baixa } \\
\text { Até } 20 \% \text { dos chefes }\end{array}$ & $\begin{array}{l}\text { RM2: Média } \\
\text { De } 20 \text { a } 50 \%\end{array}$ & $\begin{array}{l}\text { RM3: Alta } \\
\text { Mais de } 50 \%\end{array}$ \\
\hline $\begin{array}{l}\text { Renda alta } \\
\text { (acima de } 6 \text { salários mínimos) }\end{array}$ & $\begin{array}{l}\text { RA1: Baixa } \\
\text { Até } 20 \% \text { dos chefes }\end{array}$ & $\begin{array}{l}\text { RA2: Média } \\
\text { De } 20 \text { a } 50 \%\end{array}$ & $\begin{array}{l}\text { RA3: Alta } \\
\text { Mais de } 50 \%\end{array}$ \\
\hline
\end{tabular}

\section{Agrupamento dos setores}

Para o agrupamento dos setores censitários em áreas homogêneas, utilizou-se a Análise Fatorial de Correspondência (AFC) realizada no software estatístico SPAD-N (CISIA, 1998). Trata-se de uma análise multivariada, que possibilita identificar relações entre variáveis nominais e entre as suas categorias, com formação de fatores, a exemplo do que faz a Análise de Componentes Principais em variáveis ordinais (Pereira, 1999). A técnica AFC tem, sobre outras análises multivariadas, como a Análise de Kluster, a vantagem de permitir ao pesquisador que estabeleça as categorias das variáveis a partir de um modelo teórico e não da distribuição empírica dos dados. Assim, para abastecimento de água, por exemplo, a classificação em baixa, média ou alta presença não foi definida por um valor percentual obtido, mas por pontos de corte a partir dos quais as diferenças seriam importantes. Este estudo, permitiu a identificação dos setores censitários pertencentes a cada grupo homogêneo e a influência das variáveis sócio-econômicas na composição desses grupos.

A primeira análise é exploratória, e, através de uma tabela de contingência, os setores, as variáveis e suas categorias foram distribuídos em um espaço tridimensional com um centro de equilíbrio. Esse centro de equilíbrio corresponde ao ponto onde se esperava que todos estivessem localizados a um ponto médio na distribuição paramétrica. As distâncias entre os pontos observados e o centro da distribuição são medidas em $\chi^{2}$ e expressam a variabilidade da distribuição. Nas outras análises multivariadas essa distância é uma medida Euclidiana. A distribuição espacial dos setores censitários e das categorias das variáveis confirmou a tendência natural à formação de grupos distintos (Pereira, 1999).

No segundo momento, utilizou-se o método de Classificação Hierárquica Ascendente para identificação do número de grupos no conjunto e a Classificação Não Hierárquica de Partição, que permitiu a alocação estável dos setores em quatro grupos. Esse último procedimento agrupa e reagrupa os elementos do conjunto 3 a 4 vezes, até atingirem uma estabilidade espacial de seu posicionamento, para garantir a máxima homogeneidade dentro dos grupos e heterogeneidade entre eles (Crivisqui, 1997).

A utilização dessa técnica foi preferida em relação a de Componentes Principais e de 
Kluster, por dois motivos: uso de variáveis nominais e possibilidade de definição dos pontos de corte de cada categoria pelo pesquisador.

Os indicadores de desigualdade social, de maneira geral, orientam-se pelo agrupamento de variáveis que expressem as piores condições da categoria selecionada, como se pode observar nos indicadores de Jarman (1983, apud Locker, 1993), Towsend et al. (1988, apud Locker, 1993), Carstairs \& Morris (1989) e Curtis (1990). Neste estudo, optou-se por comparar resultados de duas alternativas metodológicas: uma adotando apenas as piores condições de vida, tal como preconizado na literatura, e outra utilizando todas as categorias das variáveis. A análise dos resultados demonstrou que o segundo procedimento representava, de maneira mais fidedigna, o conhecimento empírico da disposição dos setores no município.

Florianópolis tem como característica a heterogeneidade espacial da condição social, com moradores de péssimas e boas condições de vida domiciliados num mesmo espaço censitário. Poucos são os setores de péssimas ou excelentes condições de vida, exclusivamente.

\section{Resultados e discussão}

Os 297 setores censitários de Florianópolis, distribuem-se geograficamente em dez distritos: seis na região norte, dois na região sul, um na leste e um na centro-continente. Esse último distrito detém $75 \%$ da população, caracterizando uma grande concentração populacional, se considerarmos que a extensão territorial dessa região corresponde a 17,07\% do mu- nicípio. Foram excluídos seis setores que não apresentavam dados no relatório do IBGE.

A Tabela 2 apresenta as variáveis definidoras dos quatro grupos homogêneos formados pela técnica utilizada.

Observa-se que no Grupo 1, concentraramse setores censitários que apresentam excelente condição de acesso a serviços públicos: abastecimento de água, esgoto sanitário adequado e coleta de lixo; um alto índice de escolaridade; baixa taxa de analfabetismo e elevado porcentual de famílias com renda variando de 2 a 6 salários mínimos. No Grupo 2, encontramse os setores com uma condição mediana de acesso a serviços públicos e de escolaridade. $\mathrm{O}$ Grupo 3 caracteriza-se por agrupar setores com as piores condições de escolaridade e renda do município, sendo o oposto do Grupo 4, que apresenta o menor percentual de escolaridade baixa e as melhores condições de renda, com mais de $50 \%$ dos chefes de família com uma renda mensal acima de seis salários mínimos.

A partir disso, foram identificados os setores que compunham cada grupo, e sua localização no município, como pode ser visto na Figura 1 .

Analisando a Tabela 3, é possível observar que $59,8 \%$ dos setores da Região Centro, caracterizam-se por apresentar elevados percentuais de renda mensal alta dos chefes de família e reduzidos percentuais de escolaridade baixa, correspondendo a $51 \%$ do total da população na região (55.927 habitantes). Nessa região, não se encontram setores do Grupo 3, caracteristicamente de piores condições, sendo suas áreas mais precárias as dos setores incluídos no Grupo 2, com 22,1\% da população.

Tabela 2

Descrição das variáveis definidoras dos grupos.

\begin{tabular}{ll}
\hline Grupo & Definição \\
\hline 1 & AG3 - alta presença de água de abastecimento público; EG3 - alta presença \\
de esgotamento sanitário adequado; LX3 - alta presença de coleta do lixo regular; & AN1 - baixa presença de analfabetos; EA3 - alta presença de escolaridade alta; \\
& RM3 - alta presença de chefes de família com renda média. \\
& AG2 - mediana presença de água de abastecimento; EG2 - mediana presença \\
& de esgotamento adequado; AN2 - mediana presença de analfabetos; \\
& EA2 - mediana presença de escolaridade alta. \\
& LX1 - baixa presença de coleta do lixo; AN3 - alta presença de analfabetos; \\
& EB3 - alta presença de escolaridade baixa; EA1 - baixa presença de escolaridade alta; \\
& RB3 - alta presença de chefes de família com renda baixa. \\
& EB1 - baixa presença de escolaridade baixa; RB1 - baixa presença de famílias com \\
& renda baixa; RA3 - alta presença de chefes de família com renda alta. \\
\end{tabular}




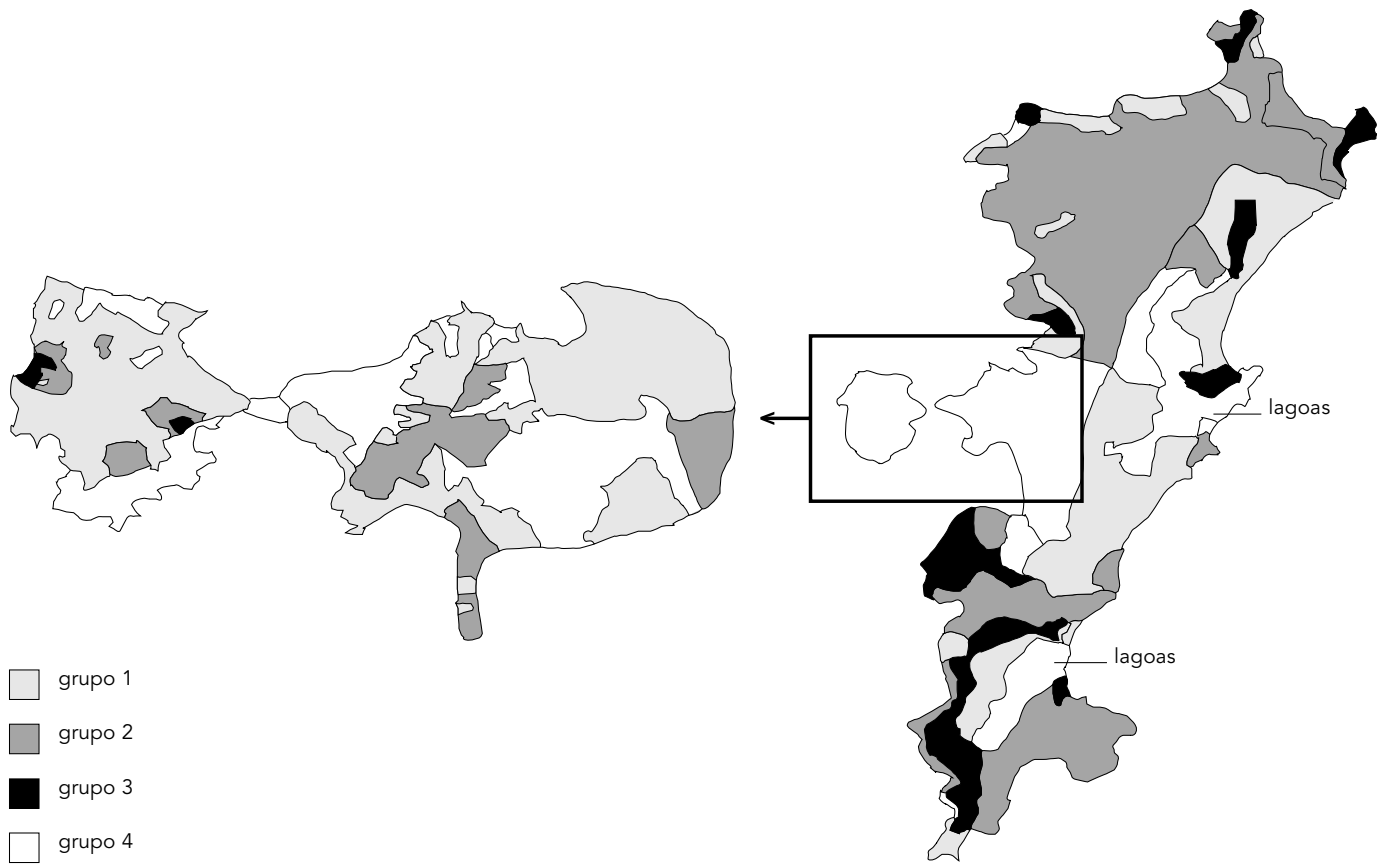

A Região Continente está majoritariamente composta $(58,7 \%)$ por setores com excelentes condições de acesso a serviços de abastecimento de água, esgoto sanitário e coleta de lixo, baixa taxa de analfabetismo e com a maioria dos chefes de família com renda mensal entre 2 e 6 salários mínimos. Nessa região, encontram-se dois setores do Grupo 3 - com piores condições - atingindo 3.519 habitantes, e 10 do Grupo 2, com 11.556 habitantes. O Grupo 4, de melhores condições de renda e escolaridade, abrange 19 setores, com 18.631 habitantes.

O Norte do município tem $95,4 \%$ da população em setores dos Grupos 1 e 2, sendo uma área de regular acesso a serviços públicos e mediana condição de escolaridade e renda. É a região que concentra o maior número de setores componentes do Grupo 3, mas correspondem a apenas $4,5 \%$ da população total da região.

$\mathrm{Na}$ Região Leste, predominam setores do Grupo 1, com 66,5\% da população. É uma região pouco populosa, com apenas 16.658 habitantes, sendo que $23,3 \%$ dessa população residem em setores classificados como de piores condições.
Por fim, a Região Sul é caracterizada pelo predomínio de setores do Grupo 2, mas com elevado percentual de setores do Grupo 3, abrangendo $48 \%$ dos setores e $12,5 \%$ da população.

Florianópolis caracteriza-se por não apresentar grandes áreas de pobreza, segundo a divisão espacial do IBGE. A observação geral dos resultados demonstra que apenas $4,3 \%$ da população reside em setores classificados como de situações precárias, e $71 \%$ em setores de boas e excelentes condições de saneamento, renda e escolaridade. É importante salientar que esse resultado não exprime o total da população em situação precária de vida, mas sim, o percentual de munícipes residentes nos setores com tais características. Se tomarmos apenas a variável renda como exemplo, veremos que, no censo de 1991, 28,3\% da população declarou renda familiar de até 2 salários mínimos. Porém, a condição de vida deve ser avaliada pela composição de indicadores sócioeconômicos, e, em Florianópolis, a distribuição heterogênea dos domicílios nos setores e a eqüidade do acesso aos serviços públicos de saneamento básico, são fatores importantes na composição de um indicador de condição de vi- 
Distribuição dos setores censitários e população, segundo agrupamento e região do Município de Florianópolis.

\begin{tabular}{|c|c|c|c|c|c|c|c|c|c|c|}
\hline \multirow[t]{3}{*}{ Região } & \multicolumn{8}{|c|}{ Grupos } & \multicolumn{2}{|c|}{ Total } \\
\hline & \multicolumn{2}{|c|}{ Grupo 1} & \multicolumn{2}{|c|}{ Grupo 2} & \multicolumn{2}{|c|}{ Grupo 3} & \multicolumn{2}{|c|}{ Grupo 4} & \multirow{2}{*}{\multicolumn{2}{|c|}{$\%$}} \\
\hline & $\mathrm{n}$ & $\%$ & $\mathrm{n}$ & $\%$ & $\mathrm{n}$ & $\%$ & $\mathrm{n}$ & $\%$ & & \\
\hline \multicolumn{11}{|l|}{ Centro } \\
\hline setores & 28 & 23,9 & 19 & 16,2 & - & - & 70 & 59,8 & 117 & 100,0 \\
\hline população & 29.533 & 26,9 & 24.210 & 22,1 & - & - & 55.927 & 51,0 & 109.670 & \\
\hline \multicolumn{11}{|l|}{ Continente } \\
\hline setores & 44 & 58,7 & 10 & 13,3 & 2 & 2,7 & 19 & 25,3 & 75 & 100,0 \\
\hline população & 48.161 & 58,8 & 11.556 & 14,1 & 3.519 & 4,3 & 18.631 & 22,8 & 81.867 & \\
\hline \multicolumn{11}{|l|}{ Norte } \\
\hline setores & 22 & 41,5 & 24 & 45,3 & 6 & 11,3 & 1 & 1,9 & 53 & 100,0 \\
\hline população & 11.609 & 40,9 & 15.468 & 54,5 & 1.276 & 4,5 & 50 & 0,2 & 28.403 & \\
\hline \multicolumn{11}{|l|}{ Leste } \\
\hline setores & 13 & 61,9 & 5 & 23,8 & 2 & 9,5 & 1 & 4,8 & 21 & 100,0 \\
\hline população & 11.080 & 66,5 & 1.170 & 7,0 & 3.875 & 23,3 & 533 & 3,2 & 16.658 & \\
\hline \multicolumn{11}{|l|}{ Sul } \\
\hline setores & 7 & 28,0 & 12 & 48,0 & 4 & 16,0 & 2 & 8,0 & 25 & 100,0 \\
\hline população & 3.956 & 21,7 & 10.338 & 56,8 & 2.268 & 12,5 & 1.654 & 9,1 & 18.216 & \\
\hline \multicolumn{11}{|l|}{ Total } \\
\hline setores & 114 & 39,2 & 70 & 24,1 & 14 & 4,8 & 93 & 32,0 & 291 & 100,0 \\
\hline população & 104.339 & 40,9 & 62.742 & 24,6 & 10.938 & 4,3 & 76.795 & 30,1 & 254.814 & \\
\hline
\end{tabular}

da mais favorável. Ou seja, a população de piores condições de vida está espalhada em setores onde predominam boas condições de vida.

\section{Conclusões}

- O estudo demonstrou a possibilidade de utilização das informações do IBGE como ferramenta importante na identificação de grupos homogêneos no município. A utilização desta técnica e a facilidade e rapidez do acesso a dados secundários, potencializam o seu uso enquanto instrumento de planejamento dos serviços.

- A metodologia utilizada permitiu a localização espacial dos setores segundo sua condição de vida e, tendo sua unidade de análise numa área compreendida em média por 300 domicílios, os setores censitários, possibilitam aos técnicos, gestores e conselheiros municipais a identificação e priorização de áreas de risco, otimizando tempo e recursos.

- Florianópolis caracteriza-se como uma cidade de bons indicadores sócio-econômicos, com baixo percentual de setores predominantemente em situação precária. Ou seja, a população em pior condição sócio-econômica vive, majoritariamente, em setores censitários de boas ou regulares condições de vida.

- As condições de vida determinam quatro grupos populacionais homogêneos, que podem nortear o planejamento dos serviços públicos, em especial a saúde, num total de 84 setores merecedores de maior atenção, dentre os quais, apenas 14, reunindo $4,3 \%$ da população, exigem prioridade máxima. As condições que melhor contribuem para esse agrupamento são: renda alta, renda baixa, escolaridade alta, analfabetismo e coleta precária do lixo.

- Quanto à distribuição regional, o Centro apresenta os melhores indicadores sócio-econômicos do município, seguido das Regiões Continente, Leste, Norte e Sul. As regiões Sul e Norte, merecem maior atenção por parte das autoridades públicas, pois seus setores concentram-se em grande parte nos Grupos de piores condições de vida. 


\section{Referências}

ALMEIDA FILHO, N. \& ROUQUAYROL, M. Z., 1992. Introdução à Epidemiologia Moderna. 2a Ed. Belo Horizonte: ABRASCO.

BORREL, C., 1997. Métodos utilizados no estudo das desigualdades sociais em saúde. In: Condições de Vida e Situação de Saúde (R. B. Barata, org.), pp. 167-195, Rio de Janeiro: ABRASCO.

CARSTAIRS, V. \& MORRIS, R., 1989. Deprivation: Explaining differences in mortality between Scotland and England and Wales. BMJ, 299:886-889.

CASTELLANOS, P. L. (org.), 1992. Sistemas Nacionales de Vigilancia de la Salud según Condiciones de Vida y del Impacto de las Acciones de Salud y Bien Estar. Washington, DC: Organización Panamericana de la Salud.

CISIA (Centre International de Statistique et d'Informatique Appliquées), 1998. SPAD Version 3. Saint Mandé: CISIA.

CRIVISQUI, E., 1997. Métodos de classificação. In: Programme de Recherche et d'Enseignement en Statistique Appliquée (E. Horber \& D. Ladiray, ed.), Manual do I Ciclo de Capacitação em Métodos Estatísticos Multivariados, pp. 23-77, Florianópolis: Universidade Federal de Santa Catarina.

CURTIS, S., 1990. Use of survey data and small area statistics to assess the link between individual morbidity and neighbourhood deprivation. Journal of Epidemiology and Community Health, 44:62-68.

FUNDAÇÃO SEADE (Fundação Sistema Estadual de Análise de Dados), 1992a. Pobreza e Riqueza: Pesquisa de Condições de Vida na Região Metropolitana de São Paulo. Educação. São Paulo: Fundação Seade.

FUNDAÇÃO SEADE (Fundação Sistema Estadual de Análise de Dados), 1992b. Pobreza e Riqueza: Pesquisa de Condições de Vida na Região Metropolitana de São Paulo. Renda. São Paulo: Fundação Seade.
FUNDAÇÃO SEADE (Fundação Sistema Estadual de Análise de Dados), 1992c. Pobreza e Riqueza: Pesquisa de Condições de Vida na Região Metropolitana de São Paulo. Habitação. São Paulo: Fundação Seade.

HORBER, E. \& LADIRAY, D., 1997. Programme de Recherche et d'Enseignement en Statistique Appliquée. Manual do I Ciclo de Capacitação em Métodos Estatísticos Multivariados. Florianópolis: Universidade Federal de Santa Catarina.

IBGE (Fundação Instituto Brasileiro de Geografia e Estatística), 1991. Censo Demográfico, 1991. Rio de Janeiro: IBGE.

JAGUARIBE, H.; SANTOS, W. G.; ABREU, M. P.; FRITSCH, W. \& ÁVILA, F. B., 1986. Brasil $2000-$ Para um Novo Pacto Social. 4a Ed. Rio de Janeiro: Paz e Terra.

LOCKER, D., 1993. Measuring social inequality in dental health services research: Individual, household and area-based measures. Community Dental Health, 10:139-150.

PAIM, J. S., 1997. Abordagens teórico-conceituais em estudos de condições de vida e saúde: Notas para reflexão e ação. In: Condições de Vida e Situação de Saúde (R. B. Barata, org.), pp. 7-30, Rio de Janeiro: ABRASCO.

PEREIRA, J. C. R., 1999. Análise de Dados Qualitativos: Estratégias Metodológicas para as Ciências da Saúde, Humanas e Sociais. 2a Ed. São Paulo: Edusp.

PEREIRA, M. G., 1995. Epidemiologia - Teoria e Prática. Rio de Janeiro: Guanabara Koogan.

Recebido em 11 de abril de 2001

Versão final reapresentada em 22 de fevereiro de 2002 Aprovado em 19 de março de 2002 